\title{
Getting to the Core of Hard Red Winter Wheat Quality
}

\author{
Adrienne Blakey \\ Plant and Soil Sciences Department \\ Faculty Sponsor: Dr. Brett Carver \\ Graduate Student Mentor: Liza Van der Laan \\ Special consultant: Dr. Patricia Rayas-Duarte
}

\begin{abstract}
As the wheat industry works to create more high-quality food products for consumers, it is necessary for plant breeders to develop new wheat varieties with improved milling and baking characteristics that maximize production efficiency while reducing dependency on vital wheat gluten or other flour additives. The purpose of this study was twofold: i) to test a popular belief that plant breeding, over time, has gradually produced varieties lacking the necessary characteristics - namely, dough strength - to allow migration from flour additives, and ii) to perform a specific assessment of gluten compression-recovery (CORE) analysis as a potential tool for wheat breeding programs to supplement industry-standard tests such as the farinograph. The farinograph and other recording dough mixers (RDMs) are used to make breeding decisions and are crucial to the success of a breeding program, a farmer's operation, and the milling and baking industries. Through this project, we determined that gluten elasticity, a key metric of CORE analysis, increased at the rate of $1.6 \%$ per breeding generation since the introduction of the heirloom variety, Turkey, and thus countering claims that higher yielding varieties lack the gluten strength once present in breeding generations long ago. A second significant finding of this project was that CORE analysis revealed differences in gluten quality among commercial wheat varieties that were more difficult to detect with more traditional, but cumbersome, flour quality tests available through the farinograph, opening up the prospect for wheat researchers to use gluten compressionrecovery analysis as a selection tool in variety development.
\end{abstract}

\section{Introduction}

Throughout the temperate zones of the world ranging from Scandinavia to Argentina and covering a wide array of climates, terrain, and elevations, wheat is one of the most important cereal grains globally as a major source of energy in the form of starch. In addition, wheat contributes protein, vitamins, dietary fiber, phytochemicals, and several other essential health benefits (Shewry \& Hey, 2015). In 2018, more than 734 million metric tons of wheat were produced globally (Shahbandeh, 2019), only to be surpassed by corn and rice, 
making wheat the third most significant crop in terms of global production. No crop plant is more widely cultivated than wheat.

All phases of wheat research, including wheat breeding, have contributed to significant gains in overall productivity, but perhaps more importantly better and more specific adaptation to multiple regions of the United States. This degree of adaptation has ensured a plentiful supply of wheat suitable for a wide variety of food products, thereby conserving the utility of the grain while extending its legacy in a changing climate and ecosphere. However, media misinformation is turning consumers away from wheat and wheat foods. The public does not view modern wheat in the same way, or with the same appreciation, as the research community. A key question addressed by this research is whether modern wheat constitutes a significant departure from heirloom varieties relative to the protein fraction present in wheat endosperm.

The wheat industry can be summarized into five groups of contributors: plant breeders, farmers, millers, bakers, and consumers. As most chains function, these groups impact the others with a simple ripple effect. The work completed by bakers affects consumers; the work of millers affects both bakers and consumers; the work of farmers affects millers, bakers, and consumers; the work of plant breeders affects the miller, bakers and consumers; and the opinions and requests of consumers affect all of the above. Over the past 100 years, an ever-increasing number of wheat varieties has been developed to increase disease and drought resistance and increase yield potential. In this effort to increase yield, plant breeders must also be conscientious of end-use value. One of the determining factors for end-use value is found in the quality and quantity of protein in the wheat kernel.

The main storage protein found in the endosperm of a wheat kernel is gluten. Analogously, storage proteins found in barley and maize are hordein and zein, respectively. Gluten is highly complex and is formed from gliadins (monomers) and glutenins (polymers) (Barbaro et al., 2018), each having unique characteristics relating to elasticity and viscosity (hence, viscoelasticity) which directly affect the usability or functionality of wheat flour for different food products. For the bread industry, the kind and amount of gluten present affects mixing tolerance and stability of the dough in a production line and, ultimately, profitability of the milling and baking industry. It is valuable to know how a flour will fare before it is placed on the production line. Within the food industry, there is increasing demand for a "clean label" from consumers. Although "clean label" is not a scientific term, it carries significant meaning to consumers looking for simple, wholesome, safe and clean food. It is not atypical for conditioners, emulsifiers, and vital wheat gluten to be added to bread products while in production. Although it is a safe way to counter inherently poor protein in the flour a baker is working with, these additives do raise concerns for consumers. This pressure from the consumers on the bakers then ripples through the millers and farmers to the breeding programs. The demand is for high quality protein. 
Industry standard RDMs, such as the farinograph, are time consuming, taking upwards of one hour to complete the analysis of one dough sample - an impossible timeframe for a wheat breeding program analyzing tens of thousands of experimental lines each year. Another standard RDM, the mixograph, is already deployed in wheat breeding research and variety development, and will continue to be that way. As the industry moves forward and works to meet growing consumer demands, it is necessary to be as efficient and accurate as possible in understanding the characteristics of wheat varieties before they are mixed into dough or put in an oven. In this study, we used the Glutomatic compressionrecovery test measuring recovery index (RI), a technology developed in 2012, to explore a more time-efficient and direct way to determine gluten quality as a potential new breeding program tool to supplement the mixograph without the time constraints of the farinograph.

The first component of this study utilized flour samples from a 100-year wheat variety panel, extending from the hard red winter (HRW) wheat ancestor, 'Turkey', to the present-day HRW variety grown on more acres than any other variety in Oklahoma, 'Gallagher'. Represented among these bookend varieties were six cycles or generations of wheat breeding. Never before has CORE testing been applied to historical breeding panels in multi-year field experiments. The first year of field experiments (2015-2016 crop season) was analyzed and reported as part of OSU's Freshman Research Scholars program. The second year of analysis was conducted under this project, thereby allowing scientific publication of the results in concert with other flour quality parameters and agronomic traits reported by the graduate advisor to this project, Liza Van der Laan.

The second component to this study was designed to survey the current germplasm base for hard winter wheat dough rheology on a breeding program-wide scale. Part of this survey was to ascertain association, if any, between farinograph metrics and CORE results. Can we learn about gluten quality from CORE testing that we may not extract from the farinograph? Can we tease out meaningful differences with CORE that do not fall out with the farinograph? Better yet, may we use our breeding resources more efficiently with CORE instead of farinograph?

Using these two different sets of wheat samples - a historical analysis and an association analysis - this review will provide insight to changes in gluten functionality throughout modern wheat breeding and the use of CORE analysis as an industry standard.

\section{Experimental Details}

Gluten samples were prepared for testing with a Glutomatic 2202 gluten washer (Perten Instruments $\mathrm{AB}$, Huddinge, Sweden) using $10 \mathrm{~g}$ white flour in a $4.8 \mathrm{~mL} 20 \% \mathrm{NaCl}$ solution. This flour suspension was mixed into a dough in the first 30 seconds, followed by additional $20 \% \mathrm{NaCl}$ water solution and mixing. The starch and excess water were drained off, and the gluten was isolated. The gluten obtained was loaded into a cylindrical shaper with a closely fitted plunger, then centrifuged in the shaper for 5 min using a Perten Centrifuge 2015 at 
$6000 \mathrm{rpm}$ (Perten Instruments AB, Huddinge, Sweden). The result was a cylindrical, pure gluten mass of about $3.5 \mathrm{~g}$ depending on the native wheat protein content (higher wheat protein content yields more gluten).

The shaped gluten samples were allowed to rest for 1 to $2 \mathrm{~min}$ before loading into the pre-calibrated Gluten CORE analyzer (Chapman, et al., 2012). The gluten samples were then subsequently compressed for $5 \mathrm{~s}$ with a peak force of $8 \mathrm{~N}$ and allowed to recover over a 55-s period. The Gluten CORE analyzer recorded the compression distance as a factor of time over a 1-min interval, from which a gluten recovery index (RI, a direct measure of gluten elasticity) was determined from three subsamples per environment per variety.

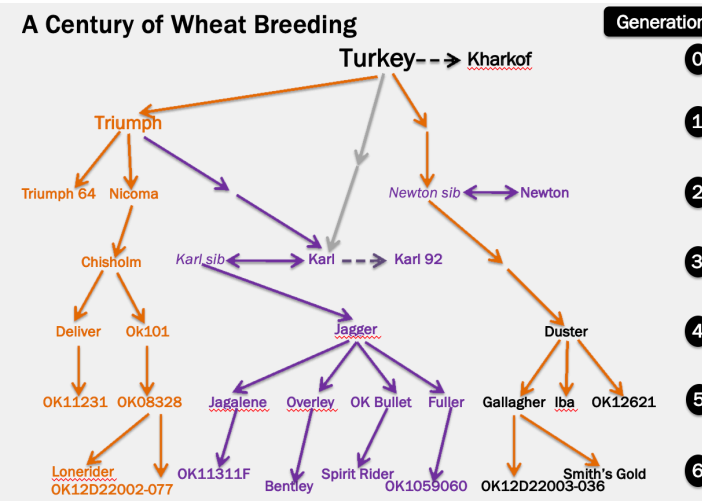

Figure 1. Southern Plains contemporary HRW wheat varieties in generation 6 trace to the HRW heirloom ancestor, Turkey, via three primary genetic lineages via Triumph, Karl, and Newton. Turkey was introduced to the Great Plains in the 1870s. Modern wheat breeding commenced in the 1920s.
In Component II, flour samples were collected from the 2017 OSU wheat variety trial conducted at the North Central Oklahoma Agronomy Station, near Lahoma, OK. This trial (http://wheat.okstate.edu/varietytesting/grain-yield-previousyrs/CR2143web2017.pdf) included 56 contemporary varieties developed by eight public and private wheat breeding programs (including OSU), encompassing the entire spectrum of modern wheat genetics in commercial production today. Farinograph analysis was conducted by the Kansas State University Hard Winter Wheat Quality
In Component I, we evaluated $30 \mathrm{HRW}$ wheat varieties spanning six generations and approximately 100 years of wheat breeding (Figure 1). Varieties were grown under optimal conditions in harvest year 2017 at Stillwater and Lahoma, OK in a randomized complete-block design with two replicates per location. This design produced 120 flour samples for Component I analysis. This 2017 data builds upon a comparable sample set from harvest year 2016. Diseases were minimized with timely application of commercially available fungicides to avoid confounding effects of differential disease resistance among varieties on flour performance.

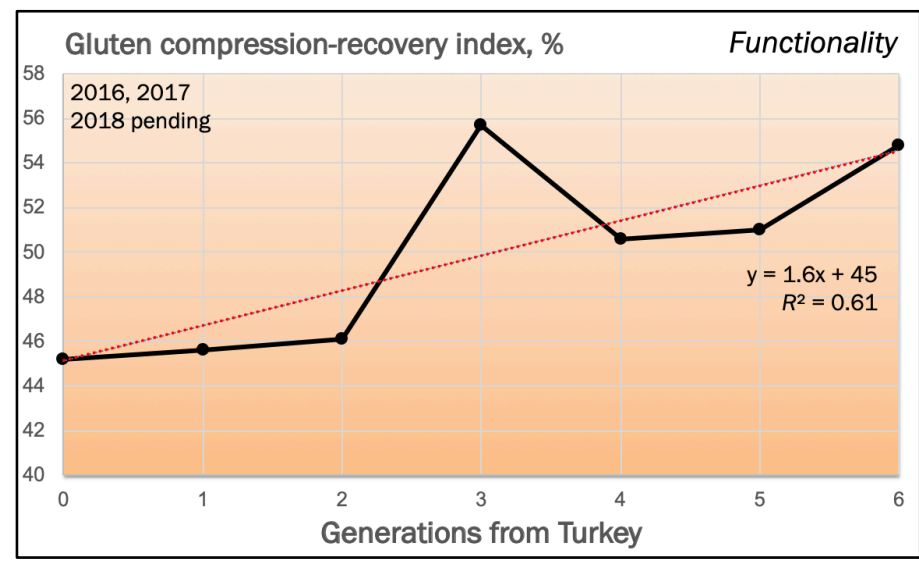

Figure 2. Gluten compression-recovery index for 120 samples from both 2016 and 2017 crop years, across six generations of wheat breeding primarily targeted for grain yield. Each data point is the mean of one or more varieties representing each generation according to Figure 1. Analysis of the 2018 harvest year samples will occur outside of this project. 
Laboratory in Manhattan, KS, using standard procedures (Wheat Marketing Center, 2004). One parameter of that analysis, farinograph stability time, was chosen for further analysis in this project, because it is the most widely used indicator of dough strength by wheat processors. Farinograph stability time measures the number of minutes a fully developed dough sample maintains maximum consistency. The same flour samples $(n=56)$ used to measure stability time were used in this project to isolate the gluten and measure CORE RI as described above.

\section{Results}

Through component I, we determined that CORE RI, in the context of gluten strength, has increased slightly during the entire modern era of wheat breeding at a rate of $1.6 \%$ per generation (Figure 2), despite the loss in total protein content of approximately one percentage unit from Turkey to current day (data not shown). While the modern wheat kernel contains by weight less protein, and thus proportionately less gluten and more starch, the elasticity and functionality of the protein has increased.

Through component II, the farinograph results did not reflect the observed variability in gluten strength as measured by CORE recovery index. Linear regression of stability time on CORE RI was not significant $(P>.05)$ (Figure 3).

\section{Discussion and Conclusions}

The Component I data reaffirmed our preliminary 2016 results. Modern wheat varieties developed in generations 3 through 6 , some of which remain in commercial production today, provide greater gluten elasticity and strength than preceding varieties. Generation 3 produced an anomalous result due to the exceptional gluten strength of the variety Karl 92, as seen in the spike in Figure 2. This result was expected, as Karl 92 is known for its exceptional dough strength. In the recent past, the wheat industry would incentivize wheat farmers to grow Karl 92 for that very reason. The graduate student advisor to this project has determined in her analysis that some varieties in generations 4 through 6 offer gluten strength not significantly different from Karl 92, but no HRW wheat variety in production today significantly exceeds Karl 92. 
As for component II, in looking to use CORE as a supplement to the mixograph or as an alternative to the farinograph, we had

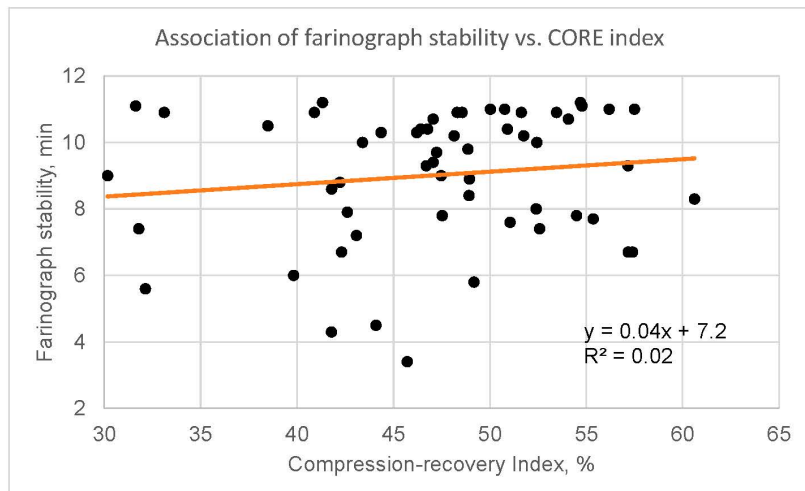

Figure 3. One industry-wide indicator of dough strength, farinograph stability, was not significantly associated with compression-recovery index, using flours produced in the 2017 OSU Wheat Variety Trials. hoped to recover information about dough strength via CORE instead of having to rely on the sluggish farinograph as another go-to instrument. As results from 2017 showed, the CORE results did not predict variability observed in dough strength measured by farinograph stability time - that is, the linear regression line in the scatter plot is relatively flat (Figure 3). This lack of fit will be pursued with additional farinograph-CORE data sets beyond this project.

One of the reasons we did not see a good fit was the year itself - 2017 environmental conditions led to data compression in farinograph stability values among this extremely diverse sample of genetics. The 2017 Great Plains wheat crop will long be remembered as one of the weakest crops the US baking industry has had to source. Therefore, stability values were lower than normally observed, and varietal differences in dough strength were much more difficult to detect using a farinograph. Normally, with this range of genetics, we expect to see stability times vary from less than 4 min to more than 20 minutes, whereas in this data set, stability time ranged from 3 to 11 minutes. Since the results of the farinograph test are used to predict processing effects, including mixing requirements for dough development, tolerance to over mixing, and dough consistency during production (Wheat Marketing Center, Inc., 2004), it is likely the CORE data is showing something different relative to gluten elasticity, one key component of dough strength.

However, one enlightening outcome of the CORE RI data was that those results did not suffer the same environmental fate or effect as farinograph stability. Data compression was not evident with a 30-percentage unit range in RI among varieties. To offer statistical perspective, least significance difference values for RI in single-site replicated experiments in Oklahoma have generally averaged 5 percentage units. The variation we observed gives breeders an opportunity to select which varieties to move forward with having desired gluten strength. 
There were some odd pairings of farinograph stability and CORE values, such that varieties known to possess desirable dough strength, whether measured by farinograph or mixograph or simply by bakers' intuition, did not produce above-average CORE values.

Examples were few among the 56variety panel, but as shown in Figure 4, 'Byrd' and 'T158' were noted as prime examples of this dissonance. With the CORE, we are working with

\begin{tabular}{|c|l|l|c|c|}
\multicolumn{1}{c}{ Entry } & Name & RI \% & Farinograph \\
\cline { 6 - 6 } 51 & 38 & Long Branch & 39.81 & 6.0 \\
52 & 37 & T158 & 38.48 & 10.5 \\
53 & 34 & LCS Wizard & 33.11 & 10.9 \\
54 & 50 & OK12D22004-016 & 32.14 & 5.6 \\
55 & 23 & SY Grit & 31.79 & 7.4 \\
56 & 40 & TAM 204 & 31.63 & 11.1 \\
57 & 43 & Byrd & 30.18 & 9.0 \\
\hline
\end{tabular}

Figure 4. Varieties such as Byrd and T158 are usually known for desirable dough strength. The farinograph results show that here, but the CORE results do not. the isolated gluten. While this is entirely appropriate given the impact of gluten on dough functionality in any food product, it is important to keep in mind other factors may determine dough properties beyond the gluten itself, such as how the starch matrix in a dough piece interacts with the gluten.

On the other hand, all varieties in the upper range of CORE RI values are well known to have desirable dough strength - OK12206-2 (release pending as 'OK Corral'), 'Brawl

\begin{tabular}{|c|c|c|c|c|}
\hline & A & B & $\mathrm{C}$ & D \\
\hline 1 & Entry & Name & $\begin{array}{c}\text { CORE RI } \\
(\%)\end{array}$ & Farinograph \\
\hline 2 & 53 & OK12206-2 & 60.61 & 8.3 \\
\hline 3 & 44 & Brawl CL+ & 57.50 & 11.0 \\
\hline 4 & 19 & SY Monument & 57.40 & 6.7 \\
\hline 5 & 3 & Ruby Lee & 57.17 & 6.7 \\
\hline 6 & 11 & WB-Cedar & 57.16 & 9.3 \\
\hline 7 & 31 & Bob Dole & 56.19 & 11.0 \\
\hline 8 & 8 & Doublestop CL+ & 55.36 & 7.7 \\
\hline 9 & 29 & Tatanka & 54.77 & 11.1 \\
\hline 10 & 47 & Smith's Gold & 54.70 & 11.2 \\
\hline
\end{tabular}

Figure 5. Varieties such as OK12206-2, Brawl CL+, SY Monument, Bob Dole, Tatanka, and Smith's Gold are known for desirable dough strength. Both the CORE and farinograph revealed this attribute.
CL+', 'SY Monument', 'Bob Dole', 'Tatanka', and 'Smith's Gold' are perfect examples of how the CORE test correctly identified the same strength the farinograph identified (Figure 5). In fact, all of those named varieties appear on most preferred variety lists published by the wheat industry. For these varieties, the CORE results clearly reflected the farinograph results with values approaching 50-60\% recovery index.

In conclusion, wheat researchers need flour analytical tools that provide consistency and maximize the connection between genetics and how we measure genetic response. This analysis gives breeders more confidence in using the CORE where we have not before. It does not enable evaluation of other flour properties critical to the industry, such as flour ash content or water absorption, but it does provide valuable insight to gluten strength.

\section{Summary}

Through this study, we observed an increase in the quality of gluten throughout the last 100 years of wheat breeding, which runs counter to the "gluten crisis" espoused by some, but 
not all, segments of the US bread industry. This allows us to be confident that certain modern varieties of wheat will be sufficient to meet the needs of high-speed baking production lines common today. Milling and baking enterprises will be required to actively search out those high-performance varieties and maintain segregation of the resulting grain throughout the supply chain. This process is just beginning to play out in the HRW wheat producing states in the form of contracted wheat production, including Oklahoma.

Another purpose of this study was to determine if gluten compression-recovery analysis is a suitable tool for wheat breeding programs to use as a supplement to standard tests and replacement for other time-inefficient tests such as the farinograph. We observed some conflicting results between the CORE and farinograph analysis. In some ways, the CORE correctly identified varieties with strong baking qualities with a high recovery index of 50 to $60 \%$. However, there were also a few varieties, typically known for good baking quality, which CORE misidentified. These varieties performed in the 30-40\% range of CORE RI, which is in contradiction to the relatively good farinograph times. With more trials from additional data set years and locations, CORE analysis will likely be used to make informative breeding decisions. Results from this project have already motivated changes in testing protocols adopted by OSU's wheat improvement team.

\section{Appendices}

\section{6a. Papers Published}

Van der Laan, L., A. Blakey, M. Tilley, and B. Carver. 2018. Adding Clarity to the Conversation about Changes in Today's Wheat. Kansas State University Plant Science Symposium. (Poster).

Carver, B.F., L. Van der Laan, A. Blakey, B. Smith, E. Lucas, M. Tilley, G. Davila el Rassi, and P. Rayas. 2019. Today's wheat: From clean record to clean label. North Dakota Nutrition Council annual meeting. 4 April 2019. Bismark, ND.

\section{6b. Acknowledgements}

I would like to thank Dr. and Mrs. Niblack for their generous sponsorship of this research scholarship. I am especially grateful to have their support for our work and my future. I would also like to extend my appreciation to my research mentor, Dr. Brett Carver. I so greatly appreciate all of his hard work in showing me the ropes to make this multi-year project a success, for believing in me as a freshman in college, and for encouraging me every step of the way. He has played a significant role in my collegiate experience and I will forever be grateful to have him as a mentor and friend. I would like to thank Liza Van der Laan for her mentorship and guidance through this entire process, but especially in helping with putting the final touches on this report and in preparing for my presentation. I am so grateful for her time and energy she has shared with me on this project. I would also like to 
thank the Oklahoma Wheat Commission for their support of the OSU wheat improvement program. Without their financial support, this research would not be possible.

\section{6c. Literature Cited}

Barbaro, M. R., Cremon, C., Stanghellini, V., \& Barbara, G. (2018). Recent advances in understanding non-celiac gluten sensitivity. National Institutes of Health.

Chapman, S. J., Mulvaney, S. J., Chinnaswamy, R., Rayas-Duarte, P., \& Allvin, B. (2012). Large deformation stress relaxation and compression-recovery of gluten representing different wheat classes. Journal of Cereal Science.

Shahbandeh, M. (2019, February 15). Wheat-Production Worldwide. Retrieved from Statista: https://www.statista.com/statistics/267268/production-of-wheat-worldwidesince-1990/

Shewry, P., \& Hey, S. (2015). The contribution of wheat to human diet and health. National Institutes of Health.

Wheat Marketing Center, Inc. (2004). Wheat and Flour Testing Methods. In A Guide to Understanding Wheat and Flour Quality (pp. 28-31). Portland. 